\title{
Mean q-Ball Strings Obtained by Constrained Procrustes Analysis with Point Sliding
}

\author{
Irina Kezele $^{1,2}$, Cyril Poupon ${ }^{1,2}$, Muriel Perrin ${ }^{3}$, Yann Cointepas ${ }^{1,2}$, \\ Vincent El Kouby $^{1,2}$, Fabrice Poupon ${ }^{1,2}$, and Jean-François Mangin ${ }^{1,2}$ \\ ${ }^{1}$ NeuroSpin, CEA, Gif-sur-Yvette, France \\ ${ }^{2}$ IFR49, France \\ ${ }^{3}$ GE Healthcare, Buc, France
}

\begin{abstract}
The idea underpinning the work we present herein is to design robust and objective tools for brain white matter (WM) morphometry. We focus on WM tracts, and propose to represent them by their mean lines, to which we associate the attributes derived from high-angular resolution diffusion imaging (HARDI). The definition of the tract mean line derives directly from the geometry of the tract fibres. We determine the fibre point correspondences and impact factors of individual fibres, upon which we estimate average HARDI models along the tract mean lines. This way we obtain a compact tract representation that exploits all the available information, and is at the same time free of the outlier influence and undesired tract edge effects.
\end{abstract}

Keywords: Tract mean-line, high-angular resolution diffusion imaging, white matter morphometry, constrained Procrustes analysis with point sliding.

\section{Introduction}

To circumvent the ill-posed problem of the inter-image alignment typically encountered in voxel-based studies of brain white matter (WM) morphometry, we adopt the concept of object-based analysis [1], and define WM entities, such as fibre tracts. In order to assess WM changes on a microscopic scale (e.g., concerning the cyto- and myelo-architectonics) [2], the attributes assigned to fibre tracts should be based on diffusion spectrum imaging or its derived methods, like high-angular resolution diffusion imaging (HARDI). For adequate representation of tracts, these attributes should remain objective, not reflecting, or at worst, reflecting only weakly the effects of deficient image resolution, like partial voluming, that is typically present at tract borders. To satisfy these demands, we put forth the idea of accentuating the information along the tract mean line (similar to $[3,4,5]$ ), and appropriately weigh the data away from the mean line. This way we lessen the edge effects while taking into consideration all the data present. The mean line that we define derives directly from the tract fibre constellation, that way exploiting the knowledge of the exact geometry of the tract's interior. The tract assigned attributes that we introduce result from HARDI, and thus bear affluent local information. HARDI data are adequately aligned and averaged inside the tract sections perpendicular to the tract mean line. This way the anisotropy estimates are representative of the overall tract volume (while rejecting the outlying points), and are enhanced by sectional averaging. 
In order to define the mean line from a bundle of tracked fibres, we need to specify a distance metric that would enable appropriate inter-fibre comparison and approximation of the ensemble average. The fibres resulting from the tracking algorithm [6], could be thought of as samples from a shape distribution around the mean shape. The deviations from the mean shape, at each related point along the fibres are expected to be small, and could be approximated by Gaussians. Together, these properties imply the employment of a shape-based metric and an analysis in the shape-space [7]. We opt for Procrustes analysis [8], similar to [9]. However, we build on the approach of [9] by incorporating certain constraints, and by implicitly allowing for some nonaffine transformations to improve the fibre point correspondence.

This paper presents the method to extract the WM fibre-tract mean-line, and to compute the average HARDI, in order to estimate the mean q-balls along the tract mean line. The goal is to setup a framework for robust tract-based WM morphometry through compact WM tract models and diminished partial voluming, while exploiting all the HARDI-based information at hand. The details and conclusions on the methods, experiments and results are given in the text that follows. Note that in this text, the term "tract" does not refer directly to an anatomical tract, but rather to a bundle of fibres that results from a tracking algorithm.

\section{Methods}

The core technique underpinning this study is Procrustes based shape analysis [10]. It is used to align the tracked fibres, to estimate the tract mean line, and to evaluate the rotations for HARDI data alignment. The overall algorithm flow is as follows. First, we place the soft-landmarks along each fibre. Initially those landmarks are equidistant. After landmark initialization, we reject the outlying fibres by first and last points dispersion, and by fibre point-distance. Then, Procrustes analysis with landmark sliding [8], point weighting, and an additional constraint to assure proper rotations [11] is employed. This results in the tract mean line, as well as the rotations for fibre alignment. The rotations are also used to turn the HARDI data onto the mean line direction. Thus transformed HARDI data are weighted by tracked fibre density, and averaged. The motivation and details for each of the aforementioned steps are given bellow.

\subsection{Landmark Placing}

In many cases of biomedical images, landmark points cannot be accurately deduced from the data [8]. With the general idea of soft point correspondence between different fibres of the same tract, we initially place the landmarks equidistantly along the fibres. Subsequent Procrustes alignment allows for landmark re-positioning depending on fibre point correspondences, while minimizing the inter-fibre spline energy. The optimal landmark number is determined empirically, and is proportional to the tract mean curvature.

\subsection{Outlier Rejection}

The probabilistic nature of the tractography algorithm [6] allows for a distribution of the tracked fibres, and thus the final tract definition may admit outliers. We employ 
filters based on fibres' start/end points dispersion, and "max-fibre distance". This way, we render the distribution of corresponding landmarks across the fibre sufficiently narrow (where the tightness of the distribution refers to linearly matched fibres [8]).

\subsection{Constrained Procrustes Analysis with Landmark Sliding}

General Procrustes analysis (GPA) [10] acts on a set of equivalent shapes, transforming them in an affine manner to minimize the square distance between the shapes, each of them represented by a set of landmarks. The procedure is iterative and is based on the ordinary Procrustes analysis (OPA) [10]. The initial step is to scale all the shapes (typically to unit size, to assure the symmetry [10]), and to align their centers of mass (commonly by translating them all to zero). Then, at each iteration we loop through the equivalence class, define the pair of shapes as a current shape $(A)$ and the average of all other shapes ( $B$ ), where $A$ and $B$ are $n x p$ matrices $(n$ - landmark number, $p$ - space dimension) that encode the coordinates of $n$ landmark points. OPA analysis assumes a least square solution of:

$$
\min \arg (R)\|A-R B\|^{2}
$$

where $R$ is the rotation matrix. It can be shown that the solution to (1) results from the singular value decomposition (SVD) $U D V^{T}$ of $A^{T} B$ ( $T$ is the matrix transpose operator, $U U^{T}=V V^{T}=I, I$ - identity matrix, $D=\operatorname{diag}\left(d_{i}\right), d_{1} \geq d_{2} \geq \ldots \geq d_{n} \geq 0$, I [11]):

$$
\begin{aligned}
& A^{T} B=U D V^{T} \\
& R=V U^{T}
\end{aligned}
$$

After all the optimal rotations for all the shapes are calculated at each iteration, the shapes are transformed accordingly, and the overall, Generalized Procrustes distance (GPD) is calculated as a sum of all square (Euclidean) distances between each pair of shapes. Iterations are repeated while the difference in two successive iterations is above a predefined threshold, or the maximum number of iterations is reached.

However, GPA can result in some improper rotations if the assumption on the small deviation from the mean shape is violated. To prevent such a degeneracy, we pose an additional constraint on rotation matrices, by inserting a sort of "sign" diagonal matrix, $S$, into the SVD solution of (3) above [11]:

$$
S= \begin{cases}I & \text { if } \operatorname{det}(U) \operatorname{det}(V)=1, \\ \operatorname{diag}(1,1, \ldots, 1,-1) & \text { if } \operatorname{det}(U) \operatorname{det}(V)=-1 \\ R=V S U^{T} & \end{cases}
$$

The point deviations for the fibre equivalent class can vary along the mean fibre. We want to assure that the points with larger spread will influence less the least square solution for optimal rotations. This is particularly important for certain tract geometries, such as in tracts that end in a "fan" shape. Thus, we insert a weighting matrix into the $A^{T} B$ term prior to $\operatorname{SVD}(2,3)$ :

$$
A^{T} W B=U D V^{T}
$$


The weighting matrix, $W$, is of a diagonal form, $W=\operatorname{diag}\left(w_{\mathrm{i}}\right)$, where $w_{\mathrm{i}}$ represent normalized weights inversely proportional to the point standard deviation. Similar SVD weighting was employed in [12], however, the weighting matrix also accounted for the case of landmark cross-correspondence. The improvement obtained by introducing the constraints on proper rotations and point-weights into the unconstrained GPA is illustrated in Fig. 1. Since the unconstrained GPA allows for reflections and assigns an equal importance to all points (irrespective of local landmark distribution across the bundle), the aligned fibres may result in a bundle that is globally not in the same position as the original bundle ("unconstrained" mean line, Fig.1.). This should be avoided, because for the subsequent HARDI averaging (Section 2.4) we need to bring the mean line to the original bundle space and the motion is not known directly. Fibre reflection might provoke a complete perturbation in inter-fibre point correspondences. The constrained GPA ("constrained" mean line, Fig.1.) results in proper interfibre point correspondences, and a bundle of aligned fibres that does not change its global position (compared to the original bundle).

While GPA acts as a sort of high-pass filter for shape differences [8], small local variations, like the ones resulting from the landmark location ambiguity, need to be addressed in complement. To accost this issue, with the main goal of resolving the inter-fibre point correspondences, we adopt the approach of Bookstein [8] and allow for a shift in landmark position, while imposing a regularity constraint in a form of 3D "thin-plate" spline energy. Landmarks are allowed to slide only along a predefined set of directions that are typically the directions towards neighbouring landmarks along the fibre, or the directions of tangents at landmark points.

The final form of our constrained GPA with landmark sliding is as follows:

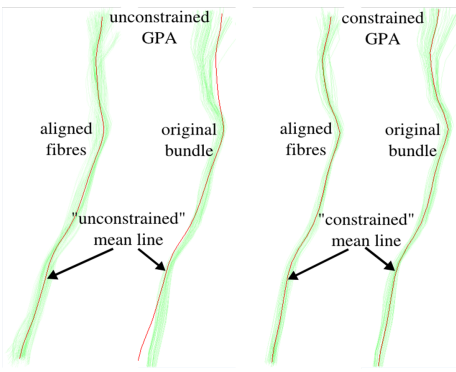

Fig. 1. Comparison between the mean line obtained by unconstrained GPA, and the mean line that resulted from the constrained Procrustes analysis

1. Choose randomly a fibre for the target for the initial linear fibre alignment and landmark relaxation

2. Apply constrained GPA on relaxed fibres

3. Perform landmark relaxation with respect to the mean fibre calculated in 2

4. Repeat 2-3 until convergence.

Typically, we employ 10 iterations for each GPA step (step 2 above). Steps 2 and 3 are commonly repeated $2-3$ times. The mean line is calculated by averaging the corresponding landmarks along the aligned fibres. 


\subsection{Average HARDI and q-Balls}

HARDI averaging is based on the assumption that HARDI signal represents a set of (independent) measurements along the prescribed directions. For each landmark point $i$ of the mean line we defined a thin cylinder $C_{i}$ with the axis parallel to the tangent at that point and of the radius superior to the maximal tract radius. All tract points $p_{i k}$ (from no matter what fibre $f_{k}$ inside the tract) that belong to a particular $C_{i}$, form a set $P_{i}$. The points $p_{i k}$ from $P_{i}$ belong to different $f_{k}$, and we can associate to them the GPA rotations $R_{k}$, related to $f_{k}$. We use these rotations to turn the HARDI data (using geodesic interpolation [15]) in voxels that correspond (by nearest neighbour interpolation) to the points $p_{i k}$. Rotated HARDI data corresponding to $p_{i k}$ of $P_{i}$ are weighted by tracked fibre density [6] in their related voxels and averaged (note that the weights are normalized prior to averaging). This results in the averaged HARDI $H_{i}$, at the mean line point $i$. Different rotations that act on $H_{i}$ in the same voxel have equal influence in the final $H_{i}$ averaging. Thus, statistically, for a large number of tracked fibres, rotation of $H_{i}$ will converge to that determined by the majority of fibres inside the voxel. Weighting by tracked fibre density prioritize HARDI data in more central voxels of the tract, while HARDI data inside edge voxels are assigned very low weights. This way we are able to use all the data, but at the same time substantially decrease the influence of the potential outliers. Consequently, we boost the power to detect and describe anisotropy inside transverse tract sections. We repeat the step of HARDI rotating and averaging for all the mean line points $i$. Upon this, at each mean line point, mean q-balls were reconstructed from the averaged $H_{i}$, by decomposing the signal to the modified spherical harmonics basis, using the algorithm described in [14].

\section{Experiments and Results}

After estimating the q-balls at every voxel of the diffusion-weighted data $(\mathrm{b}=$ $3000 \mathrm{~s} / \mathrm{mm}^{2}, 200$ directions) by Funk-Radon transform [15], a probabilistic fibre tracking algorithm [6] was performed inside the WM to track all the fibres starting at two regions of interest, defined on the entire left and right cortical grey matter surface. Fibres were clustered using the algorithm of [16].

Due to obvious space limitations, we show only a part of the results. In Fig 2, we illustrate the method on two tracts of largely different geometry: a callosal tract, and a cortico-spinal tract (of the same healthy volunteer). The final, averaged q-ball data along the mean lines of the callosal and cortico-spinal tracts are shown in Fig. 2a and b. The data are presented on the background of a RGB map of the principal diffusivities (R-x, G-y, and B-z). Note how the information on of the fibre crossing is preserved at the level of the brainstem (Fig 2. c). Figure 3 depicts the detailed results of the analysis of the generalized fractional anisotropy (GFA) along the left cingulum fascicle for a group of 5 healthy volunteers. We calculated the GFA of the q-ball models along the estimated mean lines, for the cases where we do not match/do match these mean lines by the above described Procrustes method with point sliding, this time between subjects. We wanted to assess the impact of line matching with point sliding on GFA group estimates. GFA was linearly interpolated at 15 equidistant points along the mean lines, upon which we averaged the values across the group of 


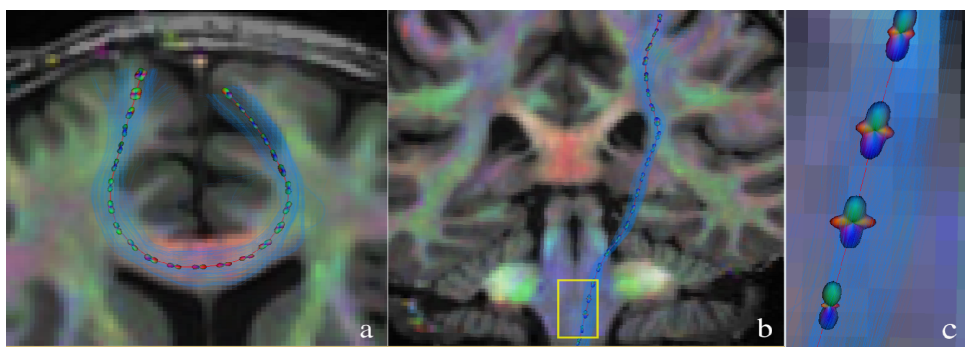

Fig. 2. Mean q-balls superimposed onto the corresponding bundles (blue), and mean-lines (red). (a) Callosal tract. (b) Cortico-spinal tract. (c) The zoom of the yellow region in b.

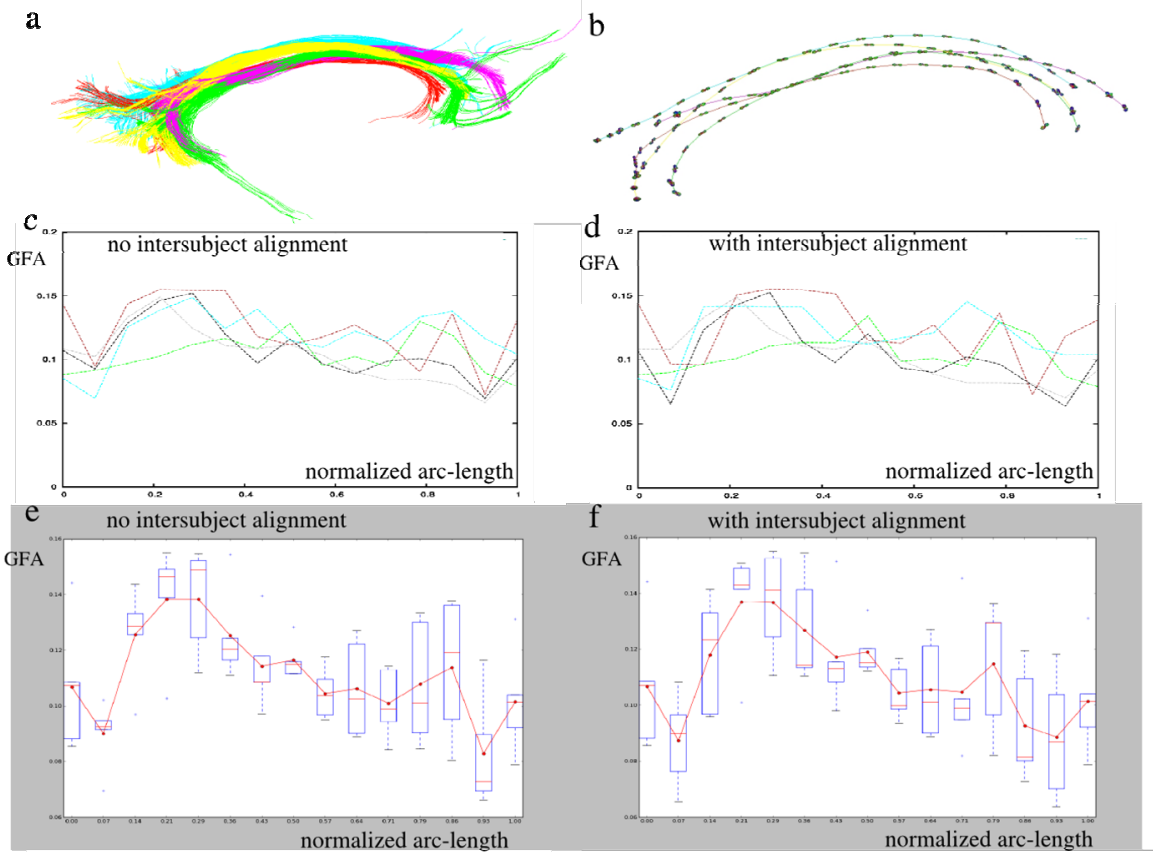

Fig. 3. Comparison of GFA estimates in "matching vs. not matching" the mean lines between subjects tests, using the constrained Procrustes method with point sliding. (a) Unfiltered fibres of the left cingulum fascicle of 5 healthy volunteers (MRIs were linearly aligned). (b) Estimated mean lines and average q-ball models along those lines. (c)-(d) GFA along the mean lines with no "sliding Procrustes" alignment , and with "sliding Procrustes" alignment. (e)-(f) The corresponding box plots of $\mathrm{c}$ and $\mathrm{d}$. (Red line connects the mean GFA values, red bar shows the median, and the lower/upper box limits correspond to lower/upper quartiles of the distribution at each point).

subjects. Despite the scarce number of the studied subjects, and for both cases (matching vs. not matching the mean lines between subjects), the inter-subject similarity in the pattern of GFA is already noticeable (Fig. 3c and d). For this small group of 
subjects, the results did not reveal any significant differences in GFA in "not matching vs. matching" tests. The idea was that while we cannot pair the first and the last points exactly due to important anatomical variability (howbeit the extreme points are also allowed to slide), point sliding should help us improve the point correspondence for the intermediate points. Although not fully conclusive (due to the small cohort size), the results raise/restate some questions: Should we match the fibres between subjects according to their shape, or rather by the attributes (e.g., GFA) attached to those fibres? How much non-linearity in fibre matching should be allowed for? The future work will comprise similar studies on larger cohorts of healthy volunteers, and with different regularization energies for matching. Hopefully, it will allow us to understand better the aforementioned problems, and to describe the distribution/patterns of GFA (and other HARDI derived attributes) with higher statistical power. This may turn into a valuable descriptor for WM morphometry studies, especially when comparing a healthy population to a population affected by a neurodegenerative or a psychiatric disorder.

\section{Conclusions}

We presented a method to robustly estimate WM tracts' mean lines, and to define the compact tract attributes derived from HARDI data. Reduced partial voluming and enhanced ability to detect and quantify anisotropy should enable unbiased tract-based WM morphometry. Other attributes could equally be included into the morphometric analysis, such as the attributes derived from the tract geometry.

\section{References}

1. Mangin, J.-F., Riviére, D., Cachia, A., Duchesnay, E., Cointepas, Y., PapadopoulosOrfanos, D., Collins, D.L., Evans, A.C., Régis, J.: Object-based morphometry of the cerebral cortex. IEEE Trans. Med. Imag. 23(8), 968-982 (2004)

2. Ashburner, J., Hutton, C., Frackowiak, R.S.J., Johnsrude, I., Price, C.J., Friston, K.J.: Identifying Global Anatomical Differences: Deformation-Based Morphometry. Human Brain Mapping 6(5), 348-357 (1998)

3. Smith, S.M., Jenkinson, M., Johansen-Berg, H., Rueckert, D., Nichols, T.E., Mackay, C.E., Watkins, K.E., Ciccarelli, O., Cader, M.Z., Matthews, P.M., Behrens, T.E.J.: Tractbased spatial statistics: Voxelwise analysis of multi-subject diffusion data. NeuroImage 31(4), 1487-1505 (2006)

4. Maddah, M., Warfield, S.K., Westin, C.-F., Grimson, W.E.L.: Probabilistic clustering and quantitative analysis of white matter fiber tracts. In: Karssemeijer, N., Lelieveldt, B. (eds.) IPMI 2007. LNCS, vol. 4584, pp. 372-383. Springer, Heidelberg (2007)

5. O'Donnell, L.J., Westin, C.-F., Golby, A.J.: Tract-based morphometry. In: Ayache, N., Ourselin, S., Maeder, A. (eds.) MICCAI 2007, Part II. LNCS, vol. 4792, pp. 160-168. Springer, Heidelberg (2007)

6. Perrin, M., Cointepas, Y., Cachia, A., Poupon, C., Thirion, B., Rivière, D., Cathier, P., El Kouby, V., Constantinesco, A., Le Bihan, D., Mangin, J.-F.: Connectivity-Based Parcellation of the Cortical Mantle Using q-Ball Diffusion Imaging. Int. J. Biomed Imaging (2008) 
7. Kendall, D.G.: Shape Manifolds, Procrustean Metrics, and Complex Projective Spaces. Bull. London Math. Soc. 16(2), 81-121 (1984)

8. Bookstein, F.L.: Landmark methods for forms without landmarks: morphometrics of group differences in outline shape. MedIA 1(3), 225-243 (1997)

9. Corouge, I., Fletcher, P.T., Joshi, S., Gouttard, S., Gerig, G.: Fiber tract-oriented statistics for quantitative diffusion tensor MRI analysis. MedI. A 10(5), 786-798 (2006)

10. Mardia, K.V., Dreyden, I.L.: Statistical shape analysis, London (1998)

11. Umeyama, S.: Least-squares estimation of transformation parameters between two point patterns. IEEE Trans. PAMI 13(4), 376-380 (1991)

12. Luo, B., Hancock, E.R.: Matching point-sets using Procrustes alignment and the EM algoritm. In: BMVC, pp. 43-52 (1999)

13. Zhang, F., Goodlett, C., Hancock, E., Gerig, G.: Probabilistic Fiber Tracking Using Particle Filtering. In: Ayache, N., Ourselin, S., Maeder, A. (eds.) MICCAI 2007, Part II. LNCS, vol. 4792, pp. 144-152. Springer, Heidelberg (2007)

14. Descoteaux, M., Angelino, E., Fitzgibbons, S., Deriche, R.: Regularized, Fast and Robust Analytical Q-Ball Imaging. Magn. Res. Med. 58(3), 497-510 (2007)

15. Tuch, D.: Q-ball imaging. Magn. Res. Med. 52, 1358-1372 (2004)

16. El Kouby, V., Cointepas, Y., Poupon, C., Rivière, D., Golestani, N., Poline, J.-B., Mangin, J.-F.: MR diffusion-based inference of a fiber bundle model from a population of subjects. In: Duncan, J.S., Gerig, G. (eds.) MICCAI 2005. LNCS, vol. 3749, pp. 196-204. Springer, Heidelberg (2005) 